\title{
THE SPIRIT III RADIOMETER DATA COMPRESSION SYSTEM
}

\author{
Scott E. Budge \\ Utah State University \\ Logan, Utah
}

\begin{abstract}
In this paper, we describe the image data compressor designed and built for the SPIRIT III radiometer which will be used to provide a real-time "quick-look" capability for the instrument. A brief description of elements of the radiometer design which determine the data compression requirements is given, followed by a description of the VQ-based hybrid data compression algorithm. Highlights of the hardware design are also discussed.

The performance of the compressor is presented. Tests made using data obtained during cold tests of the instrument indicate an RMS error of 6.26 counts for the 10:1 compression mode and 5.83 counts for the $4: 1$ mode out of a maximum of 4096 counts. The impact of focal plane nonuniformities on compression performance is also discussed.

The compressor is unique in that the VQ codebooks and Huffman codewords can be changed using an uplink capability. This capability provides the opportunity of improving the performance of the compressor over the lifetime of the spacecraft.
\end{abstract}

\section{Introduction}

The first space-based image data compression system using Vector Quantization (VQ) has been developed for the SPIRIT III radiometer built by the Space Dynamics Lab (SDL) at Utah State University (USU). The SPIRIT III instrument is designed to produce infrared (IR) images of celestial and earthlimb phenomena from an $888 \mathrm{~km}$ high sun-synchronous polar orbit. It will be launched in 1994 aboard the MSX satellite funded by the Ballistic Missile Defense Organization (BMDO), and has a mission lifetime of about 20 months [1]. The SPIRIT III data compressor is designed to compress at ratios of $4: 1$ and 10:1, which meet the compression re-

\footnotetext{
${ }^{1}$ Copyright (C)1993 by Scott E. Budge. Published by the American Institute of Aeronautics and Astronautics, Inc. with permission.
}

quirements of the earthlimb and mirror scan modes of the radiometer for real-time transmission.

The purpose of the data compressor is to provide quick-look, real-time capability for the radiometer so that health and status can be checked, and so that the correct positioning of the satellite can be confirmed. The science data gathered from the radiometer will be transmitted without compression at regular intervals in a second, larger-bandwidth channel on a delayed basis. This dual-channel transmission capability provides some redundancy, and gives a unique opportunity for comparing the lossy compressed real-time data with the delayed lossless data.

This paper discusses the design of the SPIRIT III image data compressor. Since its conceptual design in 1989, the compressor has undergone rigorous algorithmic simulation and changes, and multiple hardware design reviews and improvement. Of particular note is the need to incorporate changes into the algorithm design as the performance characteristics of the focalplane arrays were determined during focal-plane development. In addition, the design of the compressor required tradeoffs between performance and the physical constraints required by the radiometer. In Section II, we present highlights of the design of the IR radiometer and the restrictions imposed by this design on the data compression system.

In Section III, we present the compression algorithm and present performance characteristics obtained using both simulated scenes and real data from the cold tests made on the radiometer in preparation for calibration. The algorithm is based on a hybrid design which combines the high compression achievable using VQ, followed by a lossless encoder which corrects the major errors of the VQ stage while maintaining the required compression ratio. The difficulty of maintaining high quality while compressing raw (non-calibrated) focalplane data is presented and discussed.

The data compression hardware was designed to meet specific requirements for size, weight, and power, as well as the requirements for reliability and radiation hard- 
ness. In addition, an uplink capability was included to allow the compression algorithm to be improved over the life of the instrument. The hardware design is discussed in Section IV.

Finally, in Section V, we summarize and draw conclusions from the final compression system design and suggest areas for improvement in future designs.

\section{The SPIRIT III Radiometer}

The SPIRIT III radiometer is a state-of-the-art sensor designed to observe infrared phenomena in both the upper atmosphere and celestial regions. It uses existing sensor technology in its design, but is intended to be a testbed to validate the designs for future highperformance surveillance sensors. The optical and mechanical design of the instrument will not be discussed here; only the components of the design which establish the data compression requirements will be included. (For a complete discussion of the instrument design, see [1].)

The focal plane arrays (FPA) in the radiometer consist of 5 arrays of detectors, one for each IR waveband (color) of interest. The FPA is designed to operate at about $12 \mathrm{~K}$ for maximum sensitivity. Each array contains 8 columns of detectors, with each column 192 pixels long. The first four columns are grouped together as a subarray, and this subarray is offset by one half detector (pixel) width from a subarray containing the last four columns. Although the total number of detectors for the 5 colors is 7,680 , not all of the columns are used. Colors B and E use only two columns, and colors C and D use only four columns. These subsets, as well as the entire 8 columns of color A, result in a total number of 3,840 active pixels in each scan. Each pixel is quantized to 12 bits.

The radiometer is designed to operate in two different scan modes. In the first, called mirror-scan mode, a scanning mirror is used to sweep the image back and forth across the focal plane. This mode allows the instrument to continuously monitor a fixed location in space with a small revisit time. The arrays are sampled at 360 samples/s, which produces a total rate of $16.59 \mathrm{Mbps}$. The second mode, called the earthlimb mode, is a staring mode in which the scanning mirror is locked in the center of the field of regard. The FPA is sampled at 72 samples/s, resulting in a total rate of $3.32 \mathrm{Mbps}$. The image is created by the movement of the satellite in a pushbroom fashion.

The channel used by the data compressor is an Sband channel with a total bandwidth of $1 \mathrm{Mbps}$. This channel is shared with data from the SPIRIT III interferometer and some housekeeping data, which reduces the bandwidth available for compressed data to
$667 \mathrm{kbps}$. To allow for lower compression rates, the compressor only operates on 8 of the 20 columns of the FPA in mirror-scan mode (the two central columns of colors A, C, and D and the single column left-of-center of colors B and E), and 16 of the 20 columns in earthlimb mode (all 8 columns of color $\mathrm{A}$ and the two central columns of each of the other colors). The input rates to the compressor are thus reduced to $6.64 \mathrm{Mbps}$ in mirror-scan mode and $2.65 \mathrm{Mbps}$ in earthlimb mode. The required compression rates for the data compression system are 10.13:1 (mirror-scan) and 3.98:1 (earthlimb).

To reduce the weight and power of the radiometer, it was decided that it was not possible to buffer more than one sample of the FPA at a time. This meant that the data compression system could not use methods which buffer large numbers of pixels for frame-to-frame or large two-dimensional area processing. In addition, the FPA is read a column at a time, requiring processing of the image as columns of pixels.

\section{The Hybrid VQ Compression Algorithm}

The SPIRIT III data compression algorithm is a hybrid algorithm consisting of a lossy encoder followed by a lossless encoder. The entropy of a typical image from a satellite sensor is high enough to allow only about a $2: 1$ compression on the average using lossless compression techniques. Since the SPIRIT III instrument must be capable of approximately 4:1 and 10:1 compression, it becomes necessary to employ lossy compression with the highest possible fidelity. The use of a lossy vector quantization (VQ) technique allows high compression with good quality [2], which can then be followed by a lossless second stage which corrects the largest of the errors created by the lossy compression. Thus, the hybrid algorithm can maintain a guaranteed compression rate while minimizing error.

Figure 1 shows a general diagram for the hybrid encoder. In the figure, $x(m, n)$ is the input image, $\tilde{x}$ is the lossy decoded image, and $\tilde{x}-x$ is the error residual image. In this algorithm, two different compressed data streams must be multiplexed at the output and sent to the transmission or storage medium.

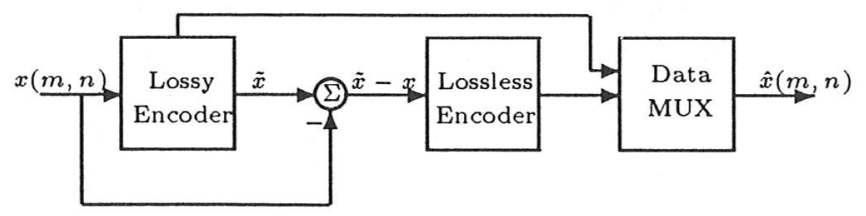

Figure 1: General diagram for a hybrid encoder. 


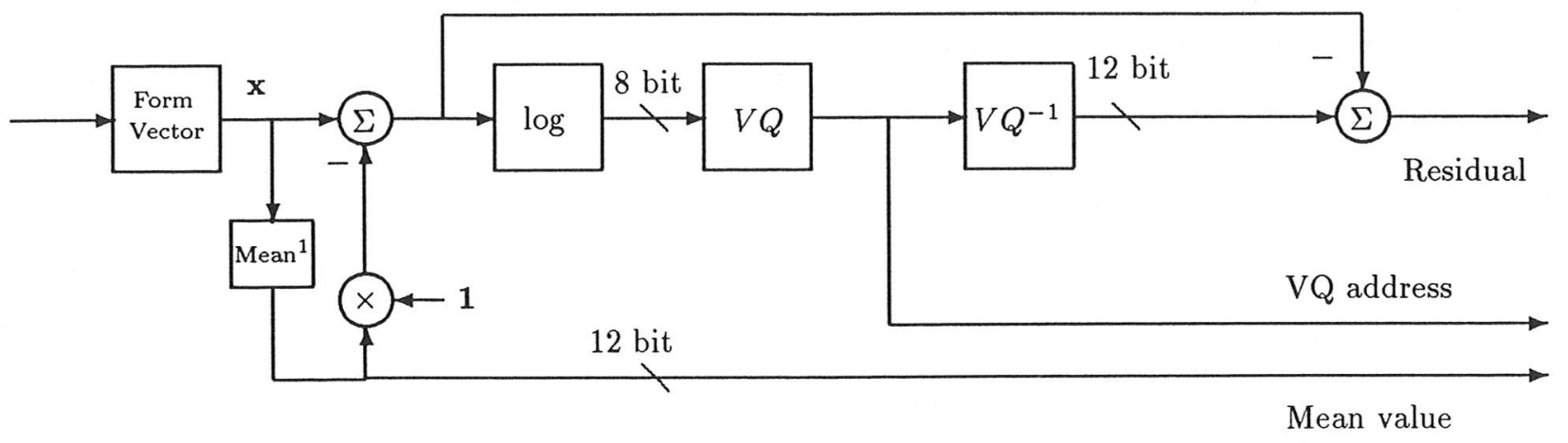

Figure 2: Flow diagram for the SPIRIT III MRVQ.

The total compression of the algorithm is determined from the compression of both the lossy and lossless encoders, and is given as

$$
C_{t o t}=\frac{1}{\frac{1}{C_{L L}}+\frac{1}{C_{V Q}}}
$$

where $C_{L L}$ is the compression of the lossless encoder and $C_{V Q}$ is the compression of the VQ encoder. Since the compression of the VQ encoder is constant for both overall compression rates, the compression of the lossless encoder is changed to achieve a 10:1 and 4:1 overall compression as described below.

\section{Mean Residual Vector Quantization}

The lossy VQ encoder algorithm is presented in Figure 2. It is based on a compression technique known as Mean Residual Vector Quantization (MRVQ) [3]. This subsection describes the MRVQ used in the algorithm.

The first step in the MRVQ algorithm is to create vectors from the image data. Each vector is made up of 16 pixel values obtained from 16x1 pixel blocks. Although Baker showed that in general square blocks perform better than columns for VQ [3], the hardware constraints imposed by the SPIRIT III instrument required that the vectors be formed from columns of pixels. Once the vectors are created, the sample mean of each vector from four adjacent segments of a column is computed and the minimum of these four mean values is subtracted from each pixel in the four vectors. The minimum mean value is then sent to a data MUX. The remaining vectors have approximately zero mean and are called mean residual vectors. It is important to note that the collection of vector means also represents a subsampled image.

The purpose of mean removal is to reduce the dynamic range requirements on the $\mathrm{VQ}$ codebook. For

\footnotetext{
${ }^{1}$ Vector sample mean is recomputed every fourth vector.
}

example, if a vector in the image has identical pixel values in each element of the vector, and another vector has equal elements with a different value, we can represent both vectors with one vector in the codebook after the means are removed. The codebook is not required to contain the average image value, but just the pixel pattern within a vector.

The VLSI chip used for performing the vector quantization requires that the pixel values be 8 -bit signed integers. It is therefore necessary to reduce the SPIRIT III 13-bit signed mean residual pixels to 8 bits (plus sign). This is done using a log lookup table which has been designed to produce a nearly constant relative error over the dynamic range of the pixels.

Each 16-pixel block (vector) of mean residual is quantized to one of 256 codebook vectors. This quantization is done by computing the distortion between the source vector and the codebook vectors using the measure

$$
d(\mathbf{x}, \mathbf{y})=\sum_{i=0}^{15}\left|x_{i}-y_{i}\right|,
$$

where $\mathbf{x}$ is the source vector and $\mathbf{y}$ is the codebook vector. The codebook vector with the minimum distortion is found and the 8-bit address in the lookup table for this vector is sent to the data MUX.

The semi-custom VLSI chip used to perform these comparisons is able to compare 16 codebook vectors to the source vector in parallel [4]. This allows the codebook to be structured in a 2-level tree with 16 vectors on the top level and 256 vectors on the bottom level (grouped as 16 children for each top level vector). The total search through the codebook can be made in two passes through the chip.

Once the minimum distortion codevector is found, the vector is then decoded from a 12-bit/element codebook and the decoded codevector is subtracted from the mean residual source vector to create a residual vector. This vector represents the distortion (or errors) caused 


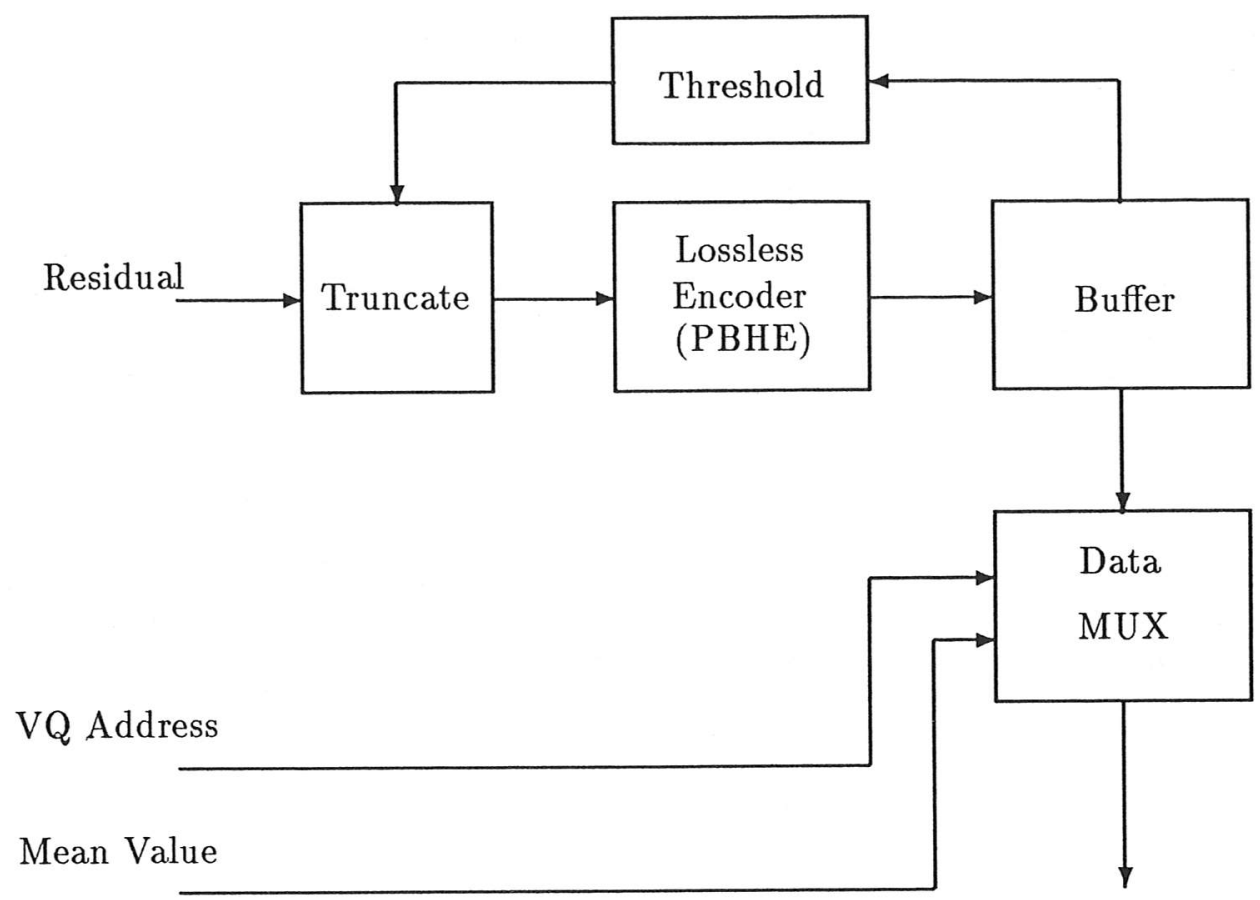

Figure 3: Flow diagram for the SPIRIT III lossless encoder.

by the MRVQ process. Ideally this vector should be close to zero, but it is well known that certain structures (such as points and edges) are not encoded well by simple VQ methods and the residual vector could have some elements with magnitudes much larger than zero. The intent of the lossless stage of the algorithm is to encode these larger errors without distortion.

\section{Lossless Residual Compression}

The second stage of the hybrid algorithm corrects the largest of the errors created by the MRVQ lossy encoding. The diagram of this stage is given in Figure 3 . Each element in the residual vector is made up of 13 bits $(12+$ sign $)$ and would require 13 bits to be stored. Entropy coding of the residual image may achieve good compression, but the compression differs for each image and is not predictable. Thus, we need to have a control mechanism to regulate the compression of the lossless stage. Regulation is done through adaptive threshold truncation.

The residual is compared pixel-by-pixel against a threshold which depends on the instantaneous fullness level of the buffer of lossless data (see Figure 3). If the threshold is exceeded, the residual value is sent to the next encoding step, otherwise 0 is sent to the next step. The image formed by these values is called the truncated residual.

We have observed that during the encoding of true images the truncated residual contains long strings of zero pixels. By using run-length encoding these strings may be greatly compressed [5] to further reduce the number of bits used by the second stage of the hybrid algorithm.

The truncated residual is thus encoded losslessly by a combination of run-length and constrained Huffman encoding. Huffman coding is a lossless technique which assigns variable length codewords to each pixel [6]. The shortest codewords are assigned to the most probable pixel values, creating the shortest average codeword length. Runs of zeros can also be assigned Huffman codewords. The combination of Huffman and runlength encoding is known as modified Huffman encoding, and is used in the FAX standard [7]. The FAX standard is based on bit-level runs instead of pixel runs. Thus, we call the coder used in this algorithm the PixelBased Huffman Encoder (PBHE).

There is a possibility that Huffman codewords may actually be longer than the data to be compressed. If the statistics used to create the Huffman codewords are not similar to the statistics of the image, it is possible that a poorly designed lossless encoder may actually expand the data.

In our lossless encoder, a constraint is used on the Huffman codewords to prevent the unwanted expansion of the data. If a low probability pixel value occurs, a short header codeword is sent, followed by the 13-bit pixel value. Thus, the longest pixel codeword possible with th SPIRIT III encoder is 17 bits long. The 
algorithm used to design the Huffman codewords with a constraint and reserved codewords was developed by Murakami [8] with modifications by Huber [9].

Encoding proceeds in real time as zeroes are grouped into runs and codewords are assigned to the runs and error residuals. Codewords are packed end-to-end and placed in an 8K-byte FIFO. The buffer is also accessed by the data multiplexor, which empties it at a constant rate. The buffer fullness level is used to establish the adaptive threshold used for truncation.

When the buffer level rises above equilibrium, the buffer feedback causes the threshold to increase so that more residual error values will be truncated to zero. The zero values are highly compressed using the Huffman codes for the runs, and the buffer fills at a slower rate. The threshold drops, and the equilibrium point is reached. The opposite occurs when the threshold drops below equilibrium.

Mean values, VQ addresses and lossless data are placed into a data frame and stored. Note that with three different data streams it is possible to have graceful degradation. If the lossless data is corrupted, the mean and VQ data can be used to create a good representation of the original image. If the VQ data is lost, an approximation to a subsampled image is still preserved.

One of the benefits of this algorithm is that post processing can be done to generate intervals that bound the error on each pixel in the image. Pixels where the residual was truncated by the adaptive threshold are in error by at most plus or minus the value of the threshold. Since the lossless buffer level can be determined by the delay between the MRVQ data and corresponding lossless data, the threshold can also be determined after encoding without sending side information if the threshold dependence on buffer level is known.

\section{Telemetry Framing}

The final step in the encoding process is to format the data into telemetry frames for the transmitter. The format for both the mirror-scan and earthlimb modes is given in Figure 4.

As shown in the figure, the telemetry stream is divided into minor frames containing 46,560 bits. A minor frame begins with a 24 bit frame sync followed by the compressed data. The number of data frames is dependent on the mode the radiometer is operating in. If the radiometer is in mirror-scan mode, there are 25 data frames per minor frame. If it is in earthlimb mode, each minor frame contains 5 data frames. Each data frame contains 48 bits of housekeeping data followed by the mean values, VQ codebook addresses, and lossless data from one sample of the FPA. Note that since the loss- less data is variable length, it may be broken into segments such that it overlaps data frames. In addition, the amount of lossless data in a data frame varies with the scan mode.

At the end of the lossless data from each sample of the array, a synchronization codeword is transmitted, followed by a frame count. The synchronization codeword is such that it cannot be confused with any possible concatenations of codewords, and is used to detect the end of the lossless data corresponding to the current sample of the FPA. The frame count is used by the decoder in the event of a loss of synchronization to correctly associate the lossless data frame with the MRVQ data frame. A 16-bit cyclic redundancy check (CRC) is also inserted by the data multiplexor. If the CRC computed by the decoder does not match, the lossless data for the current FPA sample is not decoded. This feature prevents large errors that could occur due to a misaligned truncated residual being summed with the decoded MRVQ image.

\section{Decoder}

The decoding algorithm is relatively simple when compared to the encoder. The decoder decommutates the data frames into the three parts (means, codebook entries, and lossless data) and checks the CRC to determine if the data has been corrupted. If the CRC is correct, the frame count is checked and the lossless data is decoded and added to the mean value and the vector from the decoder copy of the codebook. The complete decoded vectors are then placed in a frame buffer until the entire image is completed.

\section{Algorithm Performance}

The SPIRIT III data compressor is intended to be used for a "quick-look" at the condition of the instrument and to verify the positioning of the radiometer. Thus, the requirements for data fidelity are not stringent. We desired, however, to provide data with a RMS error comparable to the noise present in the system. In order to provide estimates of the performance of the compression system, we simulated the compressor and decompressor in software to the bit level, and ran a series of experiments on simulated star scenes to determine the true performance. The results of that simulation are given in [10], where we reported that the error introduced into the background data by the compression algorithm was comparable to the noise in the system, and the error to point sources (stars) was close to zero for normal star distributions. Similar simulations were run with earthlimb scenes with similar results.

After the hardware was built, tests were run to verify that the hardware performed as expected. These tests 


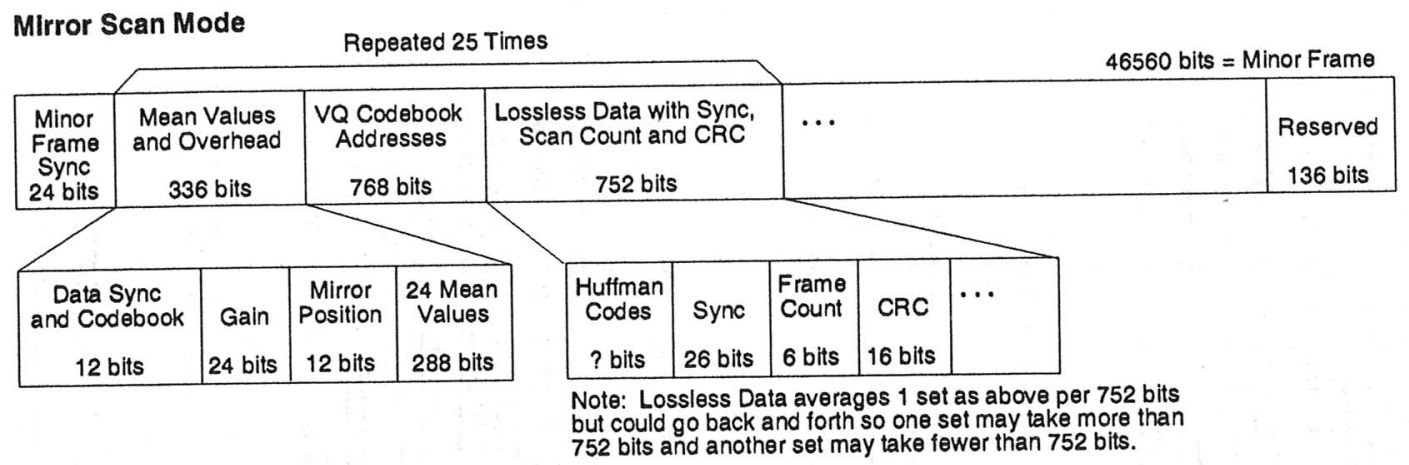

Earthlimb Mode

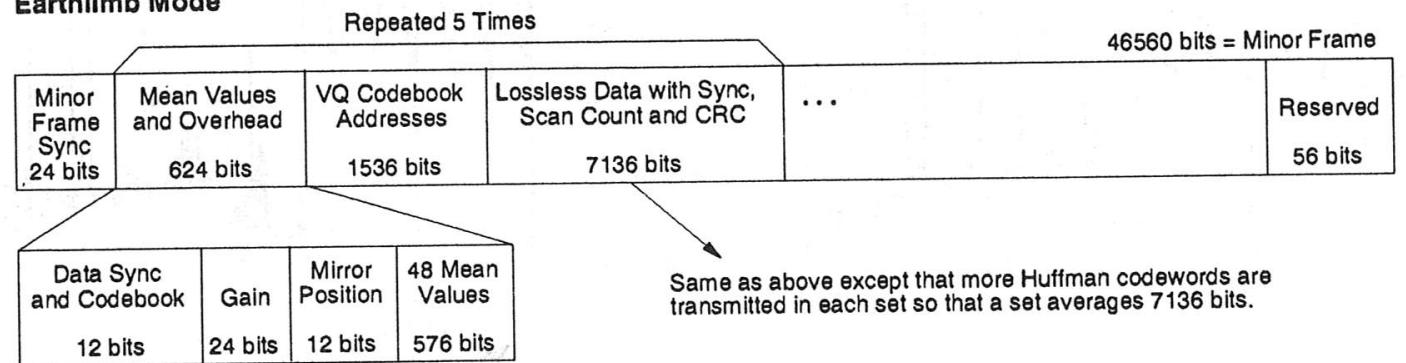

Figure 4: Telemetry frame format used for compressed data from the SPIRIT III radiometer.

showed that the hardware simulation and the hardware performed identically (except for a few small differences caused by the asynchronous data flow in the compressor hardware).

Once the SPIRIT III radiometer was completed, we continued our testing with data obtained from the instrument during the system cold tests. This data provided an unexpected result; the magnitudes of the nonuniformities of the IR FPA were more than expected. In addition, the FPA manufacturer for some of the arrays designed the arrays so that the even pixels in each column were sent through a separate signal path than the odd pixels. This difference resulted in a different offset level applied to the even and odd pixels and gave the resulting digital signal the appearance of a comb along each column. The comb effect was particularly evident for high level signals.

The effect of the nonuniformities was that the effective entropy of the source was much higher than expected. Another result was that the pixel-to-pixel correlation was lower than expected. Since the compressor was designed to use vectors created from adjacent pixels in a column, the quality of the compressed data was adversely affected.

The results from the compression of a typical column in earthlimb mode and mirror-scan mode are given in
Figures 5 and 6 , respectively.

The compressed data in the figures was obtained by processing the data with the computer simulation. It is not possible at this time to do a hardware comparison, since the current ground support equipment (GSE) will not allow the simultaneous storage of the raw data and compressed data at this time. (Modification of the GSE to do simultaneous storage is currently being considered.)

As shown in the figures, the results of the data compressor is satisfactory. The data is accurately compressed for its intended purpose and should allow the proper "quick-look" capability, thus satisfying the mission requirements for the S-band data compressor. Compression of large amounts of data from the cold tests indicate a RMS error of 6.26 counts in mirrorscan mode and 5.83 counts in earthlimb mode out of a maximum of 4096 counts.

\section{The SPIRIT III Hardware}

The data compression system for the SPIRIT III radiometer was designed to be as small as possible and to require a small amount of power. The final design required $38 \times 10$ inch $\mathrm{PC}$ boards that fit into the box which contain the other signal processing electronics. 

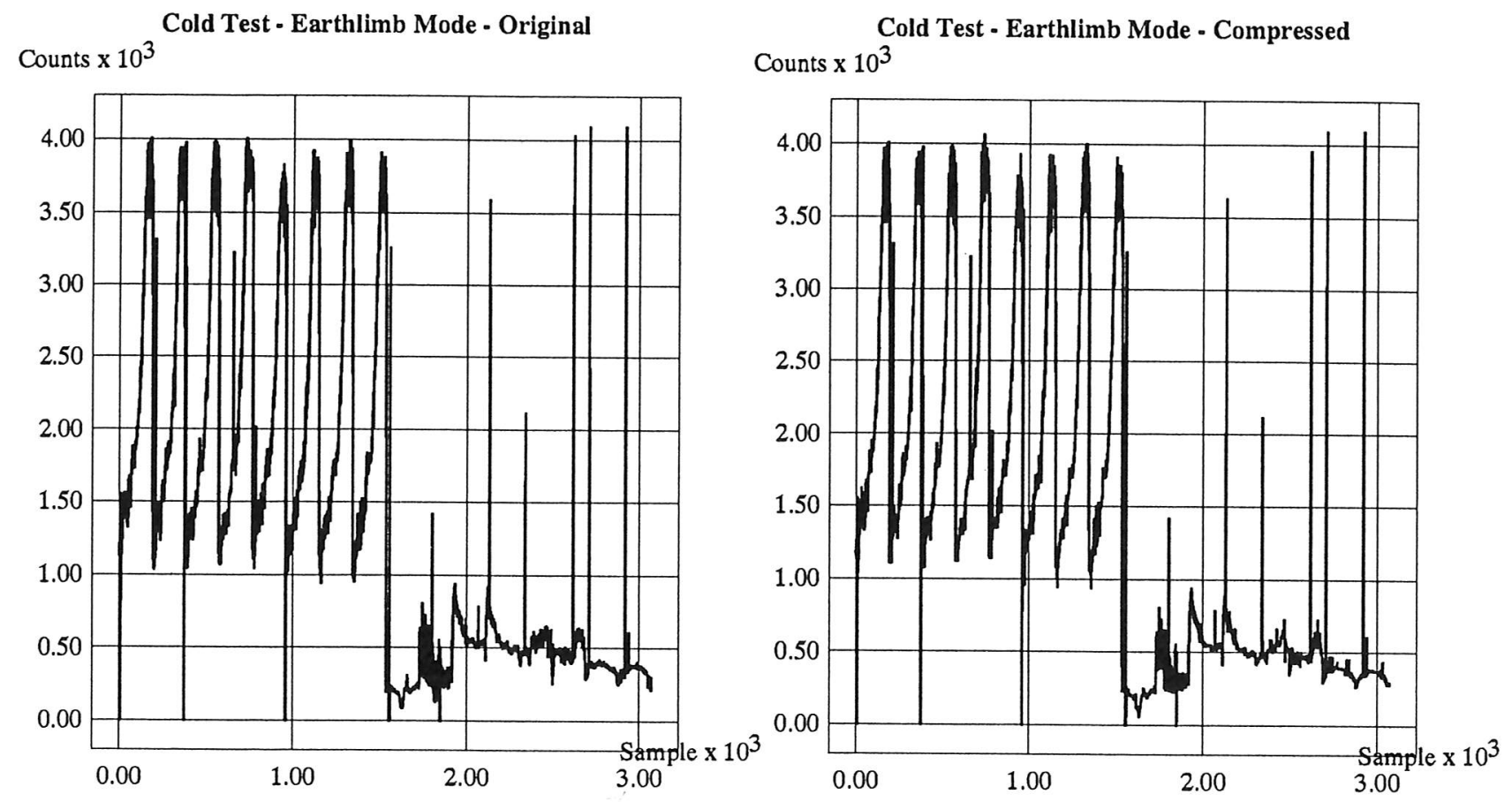

Figure 5: Results of the compression of a column of data taken during cold tests in earthlimb mode.

The physical requirements of the final design are given in Table 1.

$\begin{array}{ll}\text { Number of PC Boards } & 3 \\ \text { PC Board Size } & 8 \times 10 \text { inch } \\ \text { Weight } & 1.5 \mathrm{lbs} . \\ \text { Power Requirements } & 3 \text { watts }\end{array}$

Table 1: The SPIRIT III hardware specifications.

The heart of the data compression hardware is a semi-custom VLSI chip called the Codebook Processor Chip 16 (CPC16). This chip is used to perform the vector comparisons required by the vector quantizer. It is capable of comparing the source vector to 16 vectors from the codebook in parallel, and can be used to perform a tree search, multiple stage search, or full search of any size codebook. Table 2 lists the specifications of the CPC16.

The remainder of the compressor design is implemented in ACTEL gate arrays. These arrays are used to perform the vector mean removal, vector codebook memory control, lossless encoding, and data formatting operations. Vector codebook and Huffman codeword memory make up the rest of the hardware.

\section{Uplink Capability}

The data compressor is capable of modification during the mission due to redundant codebooks and an uplink capability. The compressor is designed with a complete set of codebook memory and Huffman codeword tables implemented using PROM, which will be loaded before launch with the best codebooks available from simulated scenes and cold test data. In addition, a complete set of codebook memory and Huffman codeword tables is implemented in EEPROM, and these codebooks can be changed during the mission to contain improved codebooks which are designed from data received during the mission. The data in the EEPROM can be uploaded to the spacecraft between critical scientific experiments and then the compressor can be switched to use the new codebooks for better performance. These redundant codebooks will also allow the compressor to function even if part of the memory is corrupted. 

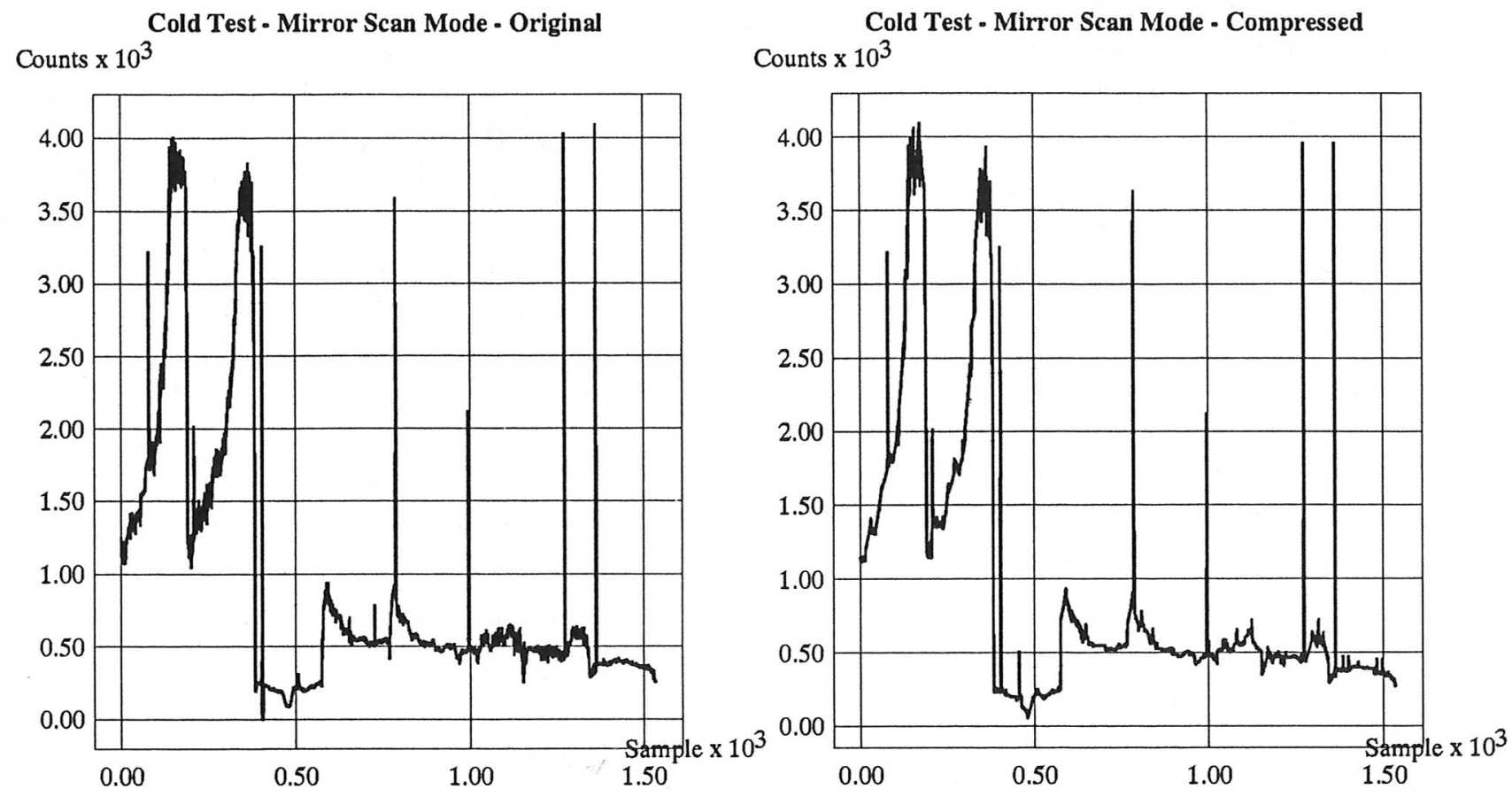

Figure 6: Results of the compression of a column of data taken during cold tests in mirror-scan mode.

\section{Summary and Conclusions}

The SPIRIT III image data compression system is currently the only space-qualified compression system which uses an algorithm based on VQ. It is designed to provide good quality data at moderate compression rates while maintaining a constant rate output for transmission in a real-time channel. It is capable of multiple compression rates $(10: 1$ and $4: 1)$ which are determined by the mode of operation of the sensor.

The algorithm used in the compressor is based on a hybrid combination of VQ and a lossless entropy encoder. The combination of the two algorithms allows the compressor to achieve the high compression needed for the radiometer data stream, while correcting the largest compression errors to improve the overall quality of the data stream.

The hardware has been tested on both synthetic images and data obtained from the cold tests of the instrument. Results of these tests indicate that the hardware performs virtually identically to the software simulation, and the encoding errors produced by the compressor are as expected.

The hardware design is capable of operating at the required 0.55 Msamples/s data rate without difficulty.
The limiting factor in performance of the hardware is the speed of the space-qualified ACTEL gate arrays and memory access time. It has been estimated that with current versions of the gate arrays, a system with a capability of greater than 2 Msamples/s can be constructed without modification to the design.

The compression system meets the design requirements for size, weight, and power. The compressor uses only about 3 watts of power to operate, which is an important consideration for a power-limited space platform.

After may tests of the algorithm, we have determined that the performance of the compression system is limited primarily by the nonuniformity of the FPAs used in the radiometer. Initial tests of the algorithm assuming uniform FPAs indicated that the algorithm was capable of about 1-2 counts RMS error on simulated star and earthlimb scenes. The data sent to the compressor by the radiometer is uncalibrated, meaning that no corrections are made for nonuniform FPA response. The FPAs finally selected for use in the instrument severely limit the quality of the data.

Our experience gained during the construction and testing of the compression system have led us to the 


$\begin{array}{ll}\text { Pixel processing time } & 38 \mathrm{~ns} \\ \text { Simultaneous comparisons } & 16 \\ \text { Maximum vector size } & 625 \text { pixels } \\ \text { Distortion measure } & \text { MAE } \\ & \text { Mean-Absolute-Error } \\ \text { Process } & 1.6 \mu \mathrm{m} \text { CMOS } \\ \text { Computational rate } & 700 \text { million } \\ & \text { add-subtract-compare } \\ & \text { operations/s @ 20 MHz } \\ \text { Power dissipation } & 0.75 \mathrm{~W} \\ & \text { @ 20 MHz } \\ \text { Qualification Level } & \text { Commercial and } \\ & \text { MIL-883B available }\end{array}$

Table 2: Specifications of the Codebook Processor Chip (CPC16).

following conclusions:

1. Based on evidence to date, the data compressor designed and implemented will provide "quick-look" capability to satisfy the S-band data requirements of the SPIRIT III mission.

2. The size of the vector codebook should be increased. We are currently investigating the use of 3-level trees which allow a choice of 4096 different code vectors.

3 . The vectors should be created in a way that reduces the impact of FPA nonuniformity on the compression method. One method of forming the vectors would be to buffer several frames of data to allow each vector to be created by successive samples of the same detector. This method has the disadvantage that correlation in time for dynamic scenes may be poor.

4. Separate codebooks could be used for each color to improve the performance of the system.

5. Tremendous increases in processing speed may be obtained by integrating the functions performed by the ACTEL gate arrays into a semi-custom VLSI chip.

We expect the experience gained during the development of the SPIRIT III compressor to be very valuable in future data compression systems.

\section{References}

[1] H. O. Ames and D. A. Burt, "Development of the SPIRIT III sensor," in Cryogenic Optical Systems and Instruments (V. R. K. Mulugin, ed.), pp. 2940, Proc. SPIE 1765, Jan. 1992.

[2] R. M. Gray, "Vector quantization," IEEE ASSP Magazine, vol. 4, pp. 12-13, Apr. 1984.

[3] R. L. Baker, Vector Quantization of Digital Images. $\mathrm{Ph} . \mathrm{D}$. dissertation, Stanford University, June 1984.

[4] P. Isralesen, "VLSI implementation of a vector quantization processor," in DCC '91 Data Compression Conference (J. A. Storer and J. H. Reif, eds.), (Snowbird, UT), p. 463, IEEE, Apr. 1991.

[5] H. Meyr, H. G. Rosdolsky, and T. S. Huang, "Optimum run-length codes," IEEE Trans. Comm., vol. COM-22, pp. 826-835, June 1974.

[6] D. A. Huffman, "A method for the construction of minimum-redundancy codes," Proc. IRE, vol. 40, pp. 1098-1101, Sept. 1952.

[7] R. Hunter and A. H. Robinson, "International digital facsimile coding standards," Proc. of the IEEE, vol. 68 , pp. 854-867, July 1980.

[8] H. Murakami, S. Matsumoto, and H. Yamamoto, "Algorithm for construction of variable length code with limited maximum word length," IEEE Trans. Comm., vol. COM-32, pp. 1157-1159, Oct. 1984.

[9] A. K. Huber, "A hybrid algorithm for compression of infrared images of space," Master's thesis, Utah State University, Logan, UT, 1993.

[10] A. K. Huber, S. E. Budge, and R. W. Harris, "Multi-rate, real time image compression for images dominated by point sources," in Space and Earth Science Data Compression Workshop, NASA Conference Publication CP-3183 (J. C. Tilton, ed.), pp. 125-136, NASA, Mar. 1992. 